\title{
Review of Facts, Data and Proposals for a Greener Internet
}

\author{
Hakim Mellah \\ GERAD and Ecole Polytechnique de Montréal \\ Montreal, Canada H3C 3A7 \\ Tel: (514) 340-4053 ext. 6021; Fax: (514) 340-5665 \\ Email: hakim.mellah@gerad.ca
}

\author{
Brunilde Sansò \\ Ecole Polytechnique de Montréal \\ Montreal, Canada H3C 3A7 \\ Tel: (514) 340-7659 ext. 4949; Fax: (514) 340-4562 \\ Email: brunilde.sanso@polymtl.ca
}

\begin{abstract}
The issue of Internet energy consumption and its contribution to global warming has been gaining importance lately. In this paper we present a compilation of facts and data extracted from scattered Internet sources and provide an overview of the basic ideas and most relevant methods suggested to reduce consumption.
\end{abstract}

\section{INTRODUCTION}

With the increase of Internet traffic, the power consumption required for routing and other functionalities has been growing accordingly [1], [2]. Energy conservation is a need, especially in most developing countries where energy is a very scarce resource [3]. Managing networks driven by the desire to protect service providers against rising energy costs while controlling the effect associated with power consumption on the environment has also the effect of decreasing operational expenditure (OPEX) and capital expenditure (CAPEX) costs [4]. In other words, designing energy efficient networks not only protects the environment but also reduces energy-related operational costs.

As a result, researchers and service providers are working hard to find solutions for the power bottleneck [1]-[10]. Different planning or operational policies have been proposed, and different ways to assess power consumption have been envisioned. The objective of this paper is to provide an overview of the existing data, basic ideas and the most relevant approaches to reduce Internet power consumption.

It is estimated that the Internet infrastructure alone currently consumes around $1 \%$ of the total electricity of broadband enabled countries [1], [2], [7]. This consumption can reach up to $8 \%$ with the inclusion of the attached equipments (servers, data centers and attached user equipments) [1], [2], [7], [11]. Not surprisingly, given the size of the Internet and the multiplicity of new personal applications, the majority of this power is consumed in the access [1], [2], dominated by data centers, routers, switches and access terminals [5], [12]. Thus, most of the proposals are driven by the necessity of reducing access energy consumption.

There are several approaches proposed to reduce energy consumption of the Internet. They can be classified into three large groups of methods: those that relate to the partition of network resources for a more effective energy utilization (virtualization), those that deal with rethinking operation and planning features to reduce energy consumption (power management and redesign) and those using and deploying new technologies in the design and fabrication of network equipments. The three approaches will be surveyed in the coming sections.

The paper is divided as follows. In Section II some facts, data and findings about energy consumption in the Internet are outlined. Virtualization techniques and approaches are presented in Section III. Section IV discusses the power management strategies to reduce energy consumption as well as appropriate methods to design or redesign the networks. Section V provides an overview about new technologies. Conclusions and recommendations are presented in Section VI.

\section{FACTS AND DATA}

Before outlining the main approaches to reduce Internet energy consumption, we review some known facts and data on the subject as they are the basic cornerstones of many of the methods being proposed. Unfortunately, there is no single outlet to extract the information or substantiate those facts with hard data. In many cases, they are of general knowledge while in other cases they are the results of very specific studies.

In what follows we have tried to provide not only the reference but also examples that can be extracted from the data reported on different outlets.

\section{A. Operation modes and consumption}

Network equipments can usually be in one of three modes: Active/busy mode, idle mode and sleep mode. While power consumption is considerably decreased in sleep mode, equipments still consume power in an idle mode when no processing or routing of the traffic occurs. What is interesting from the energy standpoint is that the consumption in idle mode is almost the same as in busy mode. An example plotted from data reported in [13] is depicted in figure 1. As it can be seen, the figure shows very little difference in power consumption when routers are idle with respect to the power consumption when they are busy.

\section{B. Type of equipment}

New equipments (those that use new and advanced technology) have a better performance versus power ratio than old 


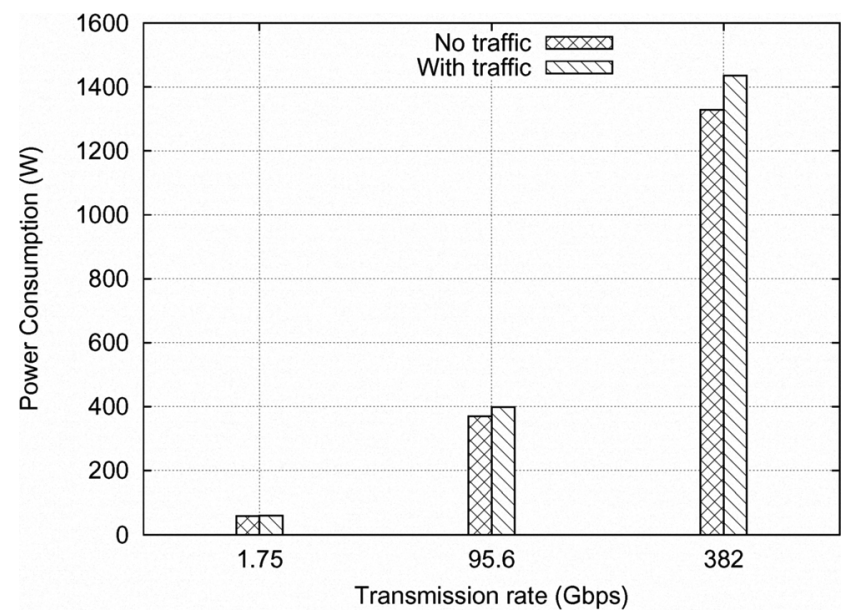

Fig. 1. Power consumption of routers with and without traffic.-Data extracted from [13].

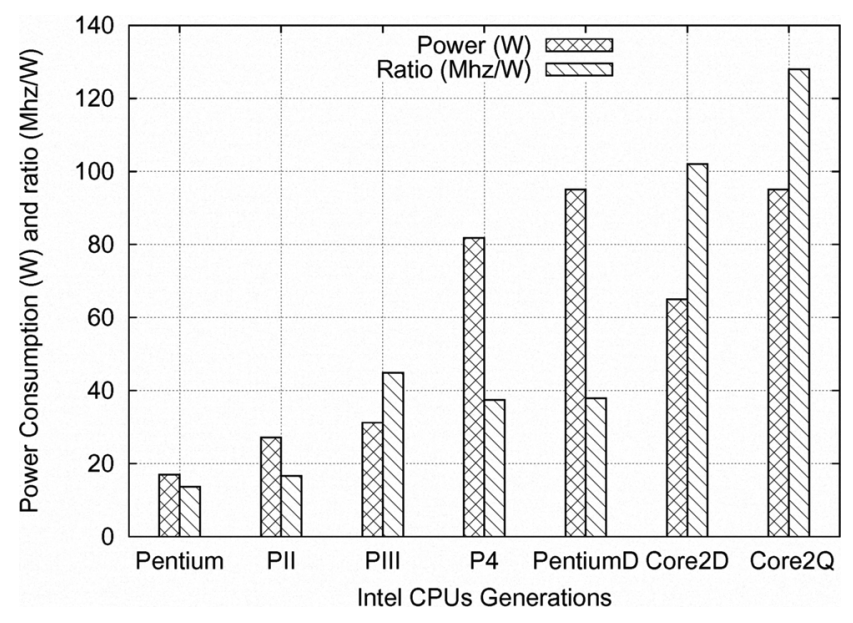

Fig. 2. Power consumption of different Intel CPU generations

ones. For instance, in figure 2 we have compiled some power and performance/power ratio for a family of Intel Pentium processors from Pentium 1 (which is based on $0.35 \mu \mathrm{m}$ technology) to the Core 2 (based on $45 \mathrm{~nm}$ technology). Most of the data was extracted from Intel's website [14]. It is interesting to note that even though the power in absolute terms increases, the ratio of performance versus power increases as well.

\section{Efficiency and equipment capacity}

Power consumption ratio in routers (which is the power budget to transmit $1 \mathrm{bps}$ ) decreases with the increase of the router's maximum throughput. This implies that, as expected, larger units are generally more power efficient. Even though this is a well known fact, we were curious to seize the amount of the difference in power ratio for different capacities and types of units. For this, we compiled the available power consumption and power ratio (Watts/Gbps) for several vendors and several types of routers with very different capacities. This was done by reviewing specifications reported in vendors' websites [15]-[19].The results are plotted in figure 3, where
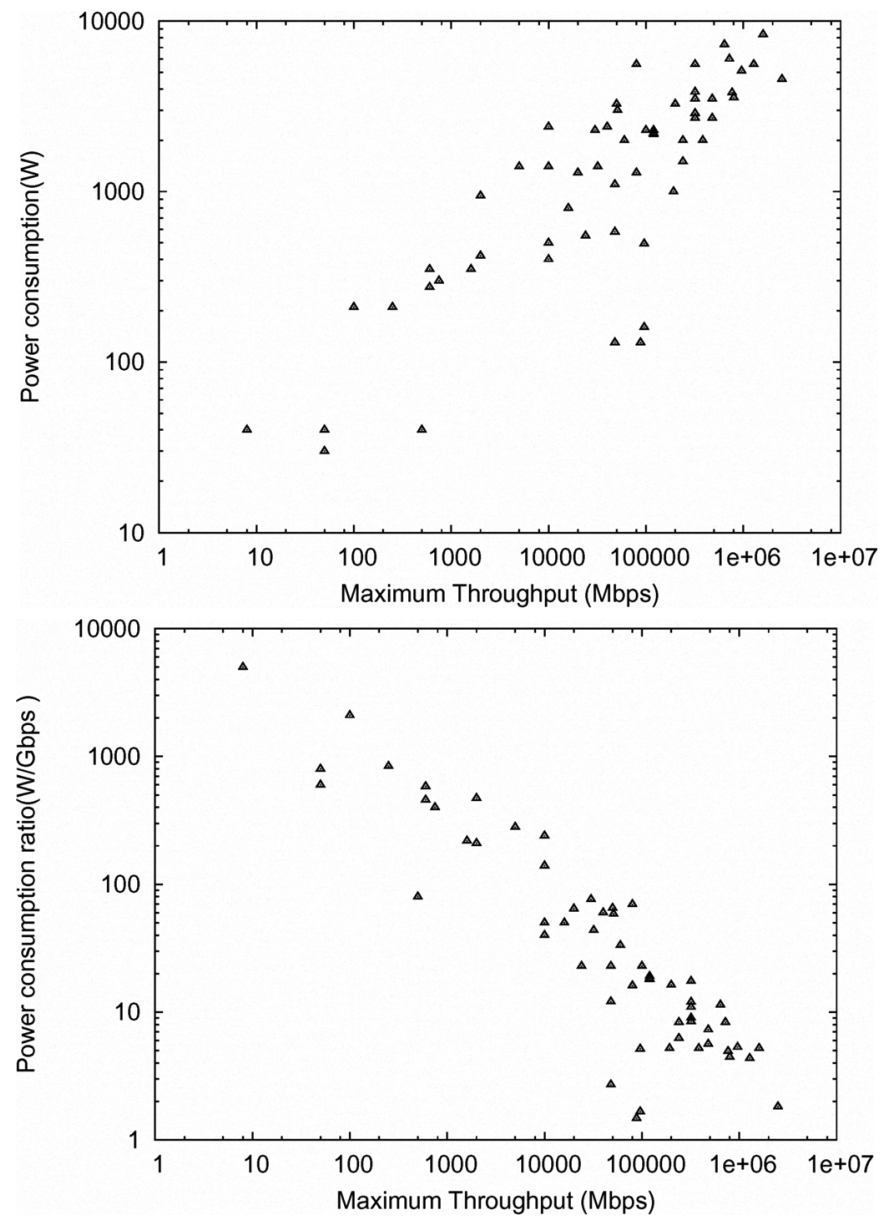

Fig. 3. Routers power consumption and consumption ratio versus maximum throughput.-Data extracted from websites [15]-[19].

it is clearly shown that even though power consumption increases with capacity and maximum throughput, router power consumption ratio definitely decreases with router's maximum throughput. The results are striking and show significant differences in terms of power consumption and efficiency for different capacities, type of routers and vendors.

\section{Packet length}

At a constant traffic rate, power consumption of routers decreases when packets length increases. This is not surprising given that for constant traffic rate, more small packets are generated, therefore more processing is required, which results in more power consumption by the router. We are not aware of any systematic testing of vendors, equipment and packet sizes. However, in [6], a set of experiments were performed on a Cisco GSR 12008 and power consumption data were presented for packets sizes of 100 byte, 576 bytes and 1500 bytes, which we plot in figure 4 . Larger packets produce a lower consumption and there is a larger difference in power consumption between large (1500 bytes) and medium packets (576 bytes) than between medium and small (100 bytes) packets. Even though the absolute value difference is small, 


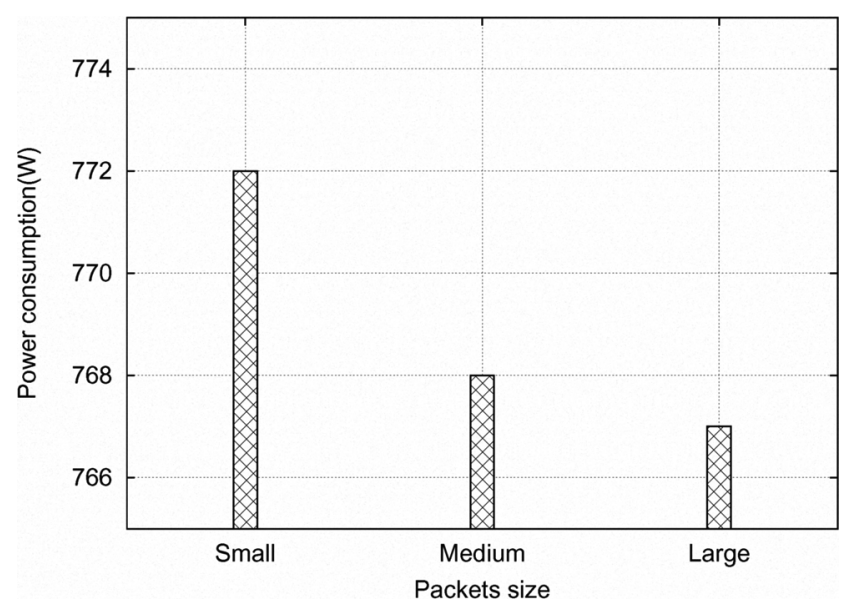

Fig. 4. Cisco GSR 12008 Router power consumption at constant traffic rate for different packet sizes. Data extracted from [6].

the results suggest a strong non-linearity with respect to packet size.

\section{VIRTUALIZATION}

For simplicity reasons, the paradigm of one-applicationone server has been widely popular among service providers and technology companies. However, devoting one server to a single application results in an significant waste of resources. With virtualization [20]-[22], it is possible to combine and consolidate different applications and execute them in a smaller number of machines. This implies less hardware and reduces the amount of energy consumption.

There exist different levels and approaches of virtualization, such as server, storage, network, platform, application, and resources virtualization [23], [24].

For example, in server virtualization, the physical server is divided into multiple virtual servers and its resources are hidden from the applications. Server virtualization can be achieved through virtual machine, paravirtualization, or using operating system virtualization [25].

In the virtual machine approach, the hardware layer of the server is virtually imitated so that each guest application is run without any modification. In addition, the hardware of the server is hidden from the guest operating system and a special hypervisor or virtual machine monitor (VMM) is needed to coordinate between the guest OS and the server hardware [25].

The paravirtualization is similar to virtual machine model, which is based on a VMM. However, the guest OS is modified or ported by the VMM [26].

Finally, in the OS Virtualization, a single OS kernel is run on the server and all the guests have to use this OS [27].

In network virtualization, available network resources and functionalities are combined in a virtual network or subdivided into virtual networks [28]. This combination aims at the optimization of the usage of the equipments internal resources to diminish overall energy consumption.
Now, despite the increasing interest in virtualization methods, they are usually compared based on performance, however, it is not clear which approach has more effect on reducing overall power consumption.

\section{Power Management AND Network Design}

In legacy networks, the parameters considered in the design were mainly network reliability, cost-effectiveness, robustness, service quality, service availability, etc. Energy consumption was most of the times neglected from design issues. With the expansion of the world wide web and the large proliferation of new applications, the Internet is becoming a hungry source of energy. Thus, power management approaches appear as a solution to explore opportunities for energy saving in current networks. Another solution is to pay careful attention to the way networks are expanded or redesigned.

\section{A. Energy saving mode}

The necessity of having network devices always on has been challenged by numerous studies [3],[29]-[42]. The idea of putting equipment to sleep exploits the fact that power consumption by devices in idle and active/busy modes are almost the same (see figure 1), and therefore putting idle devices in an energy-saving mode (asleep) reduces significantly the power consumption [13]. The general energy-saving strategy can be implemented at different levels [3] : at the individual level, by putting to sleep individual switches, routers or devices; at the network level, by combining sleep with effective on-line routing changes and bandwidth aggregation during low activity periods, so that only idle devices are put to sleep; finally, at the Internet level, by modifying the topology that allows route adaptation under a range of network loads.

The sleeping of selectively-connected devices has been the object of several studies (for instance, [29]-[31]). Sleeping hosts require an assistant or a proxy to help while in the asleep state, so many works have dealt with proxy implementation [31]-[37]. Concerning the network approaches, some studies are concerned with optimizing the choice of off-nodes and the subsequent routing (see [55] and its references). A few studies have appeared dealing with powering off some parts of wireless systems (see [56], [57] and the references therein).

\section{B. Adaptive Link Rate (ALR)}

Another power management approach that has been intensively investigated is Adaptive Link Rate (ALR), which is the ability to modify the link rate to reduce power consumption [36], [37],[43]-[46]. The technique exploits the characteristics of the network traffic in which packets are transmitted in bursts, with variable length periods of idleness or reduced link utilization between bursts. Thus, equipments may adaptively shut down or vary link rates in response to link utilization aiming at a reduced energy consumption. An example of the effectiveness that such a technique may have, can be appreciated in figure 5. The figure's data was extracted from [10] and shows the difference in power consumption of the 


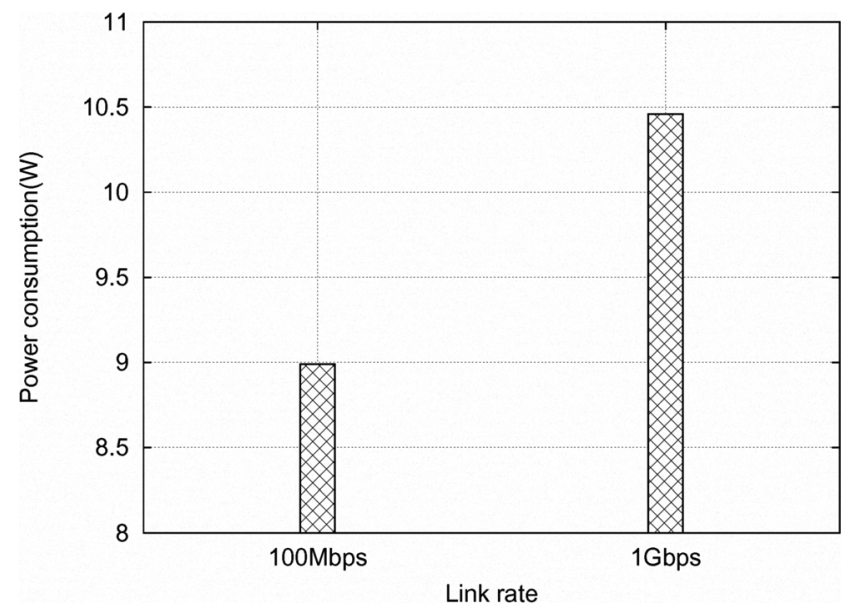

Fig. 5. Cisco Catalyst 2970 LAN switch power consumption per link for different link rates. Data extracted from [10].

Cisco catalyst 2970 LAN switch per port for 100Mbps and 1 Gbps link rates.

ALR has been adopted by IEEE as Energy Efficient Ethernet (EEE). The IEEE 802.3 EEE Study Group and the IEEE P802.3az EEE Task Force [47] are working on this technology to come out with standards that can be used by the industry. The main issues to be addressed are the coordination among nodes during the transitions from and to a low power consumption state. These transitions may result in traffic loss and the transition time should be transparent to upper layer protocols and applications.

\section{System redesign}

Taking into account power consumption when designing the system, the network and the protocols can make a significant difference in reducing energy consumption [6], [38]. This power-aware design approach can be carried out by optimizing power consumption while satisfying capacity and robustness constraints for different network users. In addition, one could think of limiting packet processing operations that require more power to only a subset of routers and developing new data link and routing protocols capable of working in an on-off network.

\section{Resilience and network consumption}

In [54] the relationship between reliability and network consumption is explored for the first time. On the one hand, the authors underline the importance of taking into account the cost of power consumption in redundant equipment and, on the other hand, present a trade-off model between resilience and power consumption in network planning exploiting the behavior of the data shown in figure 3 .

\section{NEW TECHNOLOGIES}

Adopting new technologies in the design and manufacturing of new equipments has a direct impact on their performances, including energy consumption. Amongst the most promising technologies to help reducing equipment and network energy consumptions are optical and advanced CMOS technologies.

\section{A. Optical technology}

The deployment of optical technology like IP over WDM has brought about solutions to not only bandwidth hungry applications, but also to energy efficient networks [49]. While the optical links are widely used by service providers in the backbone networks, all-optical networks that eliminate the need for electronic devices and hence O-E-O conversion are still not ready [11], [50], [51] but they are expected to bring significant reductions on energy consumption.

\section{B. Advanced CMOS technology and superconductors}

Equipments energy consumption can be reduced using advanced CMOS technology and superconductors. This is due to the fact that reducing the size of the gates, which are the building blocks of chips, results in a reduction of per-gate energy consumption. For instance, using 45-nm technology can reduce power consumption of chips by $40 \%$ compared to $65 \mathrm{~nm}$ technology [52], [53].

\section{CONCLUSIONS}

As Internet traffic increases so does the power needed for its operation, which is becoming a key environmental factor. In this paper, an overview of the most relevant data and approaches to reduce Internet energy consumption was presented.

In terms of data, there is an acute need for systematic tests and studies to determine the influence of the different equipment elements and network fonctions in overall network consumption.

Concerning energy efficiency methods we have found that although virtualization is currently very popular, other proposals such as power management and network design are gaining importance.

In the end, we believe that it is only through a combination of several approaches that we will be able to bring a significant breakthrough in energy consumption for a greener Internet.

\section{REFERENCES}

[1] J. Baliga, R. Ayre, W. V. Sorin, K. Hinton, R. S. Tucker, "Energy Consumption in Access Networks," OFC/NFOEC 2008, pp.1-3, .

[2] J. Baliga, K. Hinton, R. S. Tucker, "Energy Consumption of the Internet," COIN-ACOFT, pp.1-3, Jun 2007.

[3] M. Gupta, S. Singh, "Greening of the Internet," ACM SIGCOMM'03, pp, 19-26, Aug 2003.

[4] 1. Ceuppens, A. Sardella, D. Kharitonov, "Power Saving Strategies and Technologies in network equipment: Opportunities and Challenges, Risk and rewards," SAINT, pp. 381-384, Aug 2008.

[5] J. Baliga, R. Ayre, K. Hinton, R. S. Tucker, "Photonic switching and the energy bottleneck," International Conference on Photonics in Switching, pp.125-126, Aug 2007.

[6] J. Chabarek, J. Sommers, P. Barford, C. Estan, D. Tsiang, S. Wright, "Power Awareness in Network Design and Routing," Infocom, pp.457465, Apr 2008.

[7] K. Christensen, P. Gunaratne, B. Nordman, A. George, "The Next Frontier for Communications Networks: Power Management," Computer Communications, Vol. 27, No. 18, pp. 1758-1770, Dec 2004.

[8] K. Christensen, B. Nordman, and R. Brown, "Power Management in Networked Devices," IEEE Computer, Vol. 37, No. 8, pp. 91-93, Aug 2004. 
[9] Juniper Networks, inc. "Energy Efficiency for Network Equipment: Two Steps Beyond Greenwashing," White paper, http://www.juniper.net/ solutions/literature/white_papers/200284.pdf, Aug 2008.

[10] B. Nordman and K. Christensen, "Reducing the Energy Consumption of Network Devices," tutorial, IEEE 802 LAN/MAN Standards Committee Plenary Session, San Francisco, Jul 2005.

[11] R. S. Tucker, R. Parthiban, j. baliga, K. Hinton, R. W. A. Ayre, W. V. Sorin, "Evolution of WDM Optical IP Networks: A Cost and Energy Perspective," Journal of Lightwave Technology, vol.27, no.3, pp.243252, Feb 2009.

[12] N. McKeown, "Scaling Routers Using optics," http://yuba....stanford. edu/ nickm/talks/, Oct. 2003.

[13] — "Small Routers, L2 Switches," http://www.eccj.or.jp/top_runner/pdf/ tr_small_routers-apr_2008.pdf, Oct 2008.

[14] "Intel's website," http://www.intel.com

[15] “Cisco's website," http://www.cisco.com

[16] “Juniper's website,"http://www.juniper.net

[17] “Alaxala's website,'http://www.alaxala.com

[18] “Allied Telesis's website,"http://www.alliedtelesyn.com

[19] “3Com's website,"http://www.3com.com

[20] J. Torres, D. Carrera, K. Hogan, R. Gavalda, V. Beltran, N. Poggi, "Reducing wasted resources to help achieve green data centers," IEEE International Symposium on Parallel and Distributed Processing, IPDPS 2008. pp.1-8, Apr 2008.

[21] G. Lawton, "Powering Down the Computing Infrastructure," Computer, Vol. 40, No 2, pp. 16-19, 2007.

[22] S. Nanda and T. Chiueh, "A Survey on Virtualization Technologies," Stony Brooks University, tech. Rep. 179, pp. 1-42, 2005.

[23] G. Vallee, T. Naughton, C. Engelmann, O. Hong, S. L. Scott, "SystemLevel Virtualization for High Performance Computing," 16th Euromicro Conference on Parallel, Distributed and Network-Based Processing, PDP 2008, pp. 636-643, Feb. 2008.

[24] G. Goth, "Virtualization: Old Technology Offers Huge New Potential," IEEE Distributed Systems Online, Vol. 8, Issue 2, pp.1-4, Feb. 2007.

[25] H. Umeno, M. L. C. Parayno, K. Teramoto, M. Kawano, H. Inamasu, S Enoki, M. Kiyama, T. Aoyama, T. Fukunaga, "Performance Evaluation on Server Consolidation Using Virtual Machines," International Joint Conference SICE-ICASE, 2006, pp.2730-2734, Oct. 2006.

[26] V. Chaudhary, C. Minsuk, J. P. Walters, S. Guercio, S. Gallo, "A Comparison of Virtualization Technologies for HPC," 22nd International Conference on Advanced Information Networking and Applications, AINA 2008, pp. 861-868, Mar. 2008.

[27] D. G. Kim, S. M. Lee, D. R. Shin, "Design of the Operating System Virtualization on L4 Microkernel," 4th International Conference on Networked Computing and Advanced Information Management, NCM '08, pp. 307-310, Vol. 1, Sept. 2008.

[28] J. S. Turner, D. E. Taylor, "Diversifying the Internet," IEEE Global Telecommunications Conference, GLOBECOM '05, pp. 755-760, Vol. 2, Dec. 2005.

[29] L. Irish and K. Christensen, "A 'Green TCP/IP' to Reduce Electricity Consumed by Computers," Proceedings of IEEE Southeastcon, pp. 302 305, Apr 1998.

[30] M. Allman, K. Christensen, B. Nordman, V. Paxson, "Enabling an Energy-Efficient Future Internet Through Selectively Connected End Systems," Sixth Workshop on Hot Topics in Networks (HotNets-VI), Nov 2007.

[31] M. Jimeno, K. Christensen, and B. Nordman, "A Network Connection Proxy to Enable Hosts to Sleep and Save Energy," The IEEE International Performance Computing and Communications Conference, IPCCC'08, pp.101-110, Sep 2008.

[32] M. Jimeno and K. Christensen, "A Prototype Power Management Proxy for Gnutella Peer-to-Peer File Sharing," Proceedings of the IEEE Conference on Local Computer Networks, pp. 210-212, Oct 2007.

[33] M. Jimeno, K. Christensen, and A. Roginsky, "A Power Management Proxy with a New Best-of-N Bloom Filter Design to Reduce False Positives," Proceedings of the IEEE International Performance Computing and Communications Conference, pp. 125-133, Apr 2007.

[34] P. Purushothaman, M. Navada, R. Subramaniyan, C. Reardon, and A. George, "Power-Proxying on the NIC: A Case Study with the Gnutella File-Sharing Protocol", Proceedings of the IEEE Conference on Local Computer Networks, pp. 519-520, Nov 2006.

[35] J. Klamra, M. Olsson, K. Christensen, and B. Nordman, "Design and Implementation of a Power Management Proxy for Universal Plug and Play," Proceedings of the Swedish National Computer Networking Workshop (SNCW 2005), Sep 2005.

[36] C. Gunaratne and K. Christensen, "Ethernet Adaptive Link Rate: System Design and Performance Evaluation", Proceedings of the IEEE Conference on Local Computer Networks, pp. 28-35, Nov 2006.

[37] C. Guratne, K. Christensen, and B. Nordman, "Managing Energy Consumption Costs in Desktop PCs and LAN Switches with Proxying, Split TCP Connections, and Scaling of Link Speed,' International Journal of Network Management, Vol. 15, No. 5, pp. 297-310, Sep/Oct 2005.

[38] ITU-T, "Next-Generation Networks and Energy Efficiency," ITU-T Technology Watch Briefing Report Series, No. 7, Nov. 2007

[39] K. Sabhanatarajan, A. Gordon-Ross, M. Oden, M. Navada, and A. George, "Smart-NICs: Power Proxying for Reduced Power Consumption in Network Edge Devices," IEEE Computer Society Annual Symposium on VLSI (ISVLSI), Apr 2008.

[40] C. Gunaratne, and K. Christensen, "A New Predictive Power Management Method for Network Devices," IEE Electronics Letters, Vol. 41, No. 13, pp. 775-777, Jun. 2005.

[41] K. Christensen, "The Next Frontier for Communications Networks: Power Management," Proceedings of SPIE - Performance and Control of Next-Generation Communications Networks, Vol. 5244, pp. 1-4, Sep 2003.

[42] K. Christensen and F. Gulledge, "Enabling Power Management for Network-Attached Computers," International Journal of Network Management, Vol. 8, No. 2, pp. 120-130, Mar.-Apr. 1998.

[43] B. Zhang, K. Sabhanatarajan, A. Gordon-Ross, and A. George, "RealTime Performance Analysis of Adaptive Link Rate," the IEEE Conference on Local Computer Networks, pp.282-288, Oct. 2008.

[44] C. Gunaratne, K. Christensen, S. Suen, and B. Nordman, "Reducing the Energy Consumption of Ethernet with an Adaptive Link Rate (ALR)," IEEE Transactions on Computers, Vol. 57, No. 4, pp. 448-461, Apr 2008.

[45] C. Gunaratne, K. Christensen, and S. Suen, "Ethernet Adaptive Link Rate (ALR): Analysis of a Buffer Threshold Policy," Proceedings of IEEE GLOBECOM, Nov 2006.

[46] H. Anand, C. Reardon, R. Subramaniyan, and A. George, "Ethernet Adaptive Link Rate (ALR): Analysis of a MAC Handshake Protocol," Proceedings of the IEEE Conference on Local Computer Networks, pp. 533-534, Nov 2006.

[47] "IEEE 802.3 Energy Efficient Ethernet Study Group website,"http: //grouper.ieee.org/groups/802/3/eee_study/index.html

[48] B. Sansò and H. Mellah, "On Reliability, Performance and Internet Power Consumption," to appear in the Proceedings of DRCN 09, washington DC, Oct. 2009.

[49] Y. B.Choi, J. Y. Kim, K. Y. Kim, "Wavelength-Division Multiplexed Optical Packet Networks for TCP-IP Backbone," IEEE TENCON, pp.493-497, 2000.

[50] T. Asami, S. Namiki, "Energy Consumption Targets for Network Sytems," ECOC 2008, vol. 2, pp. 147-150, Sep. 2008.

[51] A. Gladisch, C. Lange, R. Leppla, "Power Efficiency of Optical Versus Electronic Access Networks," ECOC 2008, vol. 2, pp. 143-146, Sep. 2008.

[52] G. I. Papadimitriou, C. Papazoglou, A. S. Pomprtsis, "Optical Switching: Switch fabrics, techniques, and architectures," IEEE Journal of Lightwave Technology, 21(2), pp.384-405, 2003.

[53] "Texas Instruments website," http://www.ti.com/research/docs/index. shtml

[54] B. Sansò and H. Mellah, "On Reliability, Performance and Internet Power Consumption," to appear in Proceedings of DRCN 09, Washington DC, October 2009.

[55] L. Chiaravigli, M. Mellia and F. Neri, "Reducing Power Consumption in Backbone Networks," in Proceedings of IEEE ICC2009, June 2009.

[56] M. Ajmone-Marsan, L. Chiaraviglio, D. Ciullo, M. Meo, "Optimal Energy Savings in Cellular Access Networks," in Proceedings of GreenComm 1, June 2009.

[57] A. Jardosh, K. Papagiannaki, E.M. Belkin, K. C. Almeroth, G. Iannaccone and B. Vinnacota, "Green WLAN:On Demand WLAN Infrastructures," To appear in Mobile Networks and Applications (MONET) Journal special issue on Recent Advances in IEEE 802.11 WLANs, 2009. 